\title{
Schwarz Waveform Relaxation for Heat Equations with Nonlinear Dynamical Boundary Conditions
}

\author{
Shu-Lin Wu \\ School of Science, Sichuan University of Science and Engineering, Zigong, Sichuan 643000, China \\ Correspondence should be addressed to Shu-Lin Wu; wushulin84@hotmail.com
}

Received 4 July 2013; Revised 17 November 2013; Accepted 1 December 2013

Academic Editor: Changbum Chun

Copyright ( 2013 Shu-Lin Wu. This is an open access article distributed under the Creative Commons Attribution License, which permits unrestricted use, distribution, and reproduction in any medium, provided the original work is properly cited.

\begin{abstract}
We are interested in solving heat equations with nonlinear dynamical boundary conditions by using domain decomposition methods. In the classical framework, one first discretizes the time direction and then solves a sequence of state steady problems by the domain decomposition method. In this paper, we consider the heat equations at spacetime continuous level and study a Schwarz waveform relaxation algorithm for parallel computation purpose. We prove the linear convergence of the algorithm on long time intervals and show how the convergence rate depends on the size of overlap and the nonlinearity of the nonlinear boundary functions. Numerical experiments are presented to verify our theoretical conclusions.
\end{abstract}

\section{Introduction}

Schwarz waveform relaxation (SWR) algorithm can be regarded as a combination of the Schwarz domain decomposition (DD) method $[1,2]$ and the waveform relaxation method $[3,4]$. It is characterized by first partitioning the spatial domain into several subdomains and then solving subproblems simultaneously inside each subdomain through iterations (see [5-7] for the original idea of this approach). In the classical DD framework, one first discretizes the interested problem in time direction by using an implicit time integrator and then solves the state steady problems by using the DD iterative process. Therefore, compared to the classical DD approach, the SWR algorithm possesses the advantages that to be able to treat different subdomains numerically differently with an adapted procedure for each subdomain.

The SWR algorithm has been studied widely and deeply by many authors during the past decade. For example, Gander and his colleagues have investigated the convergence of the algorithm for linear heat equations $[5,8]$, for the linear (advection) reaction diffusion equation [9-11]. The nonlinear problems have also been investigated; see [6] for the reaction diffusion equation with a nonlinear reaction term and [12] for the nonlinear conservation laws. Nowadays, the SWR algorithm has been becoming more and more popular in scientific and engineering computing and has been adopted to solve complex problems arising from physics and engineering. For example, Martin [13] applied the algorithm to the shallowwater problem; Dolean et al. [14] and El Bouajaji et al. [15] investigated the application of the algorithm for the Maxwell's equations and Zhang and Jiang [16] applied the algorithm to time-periodic parabolic problems.

However, all of the aforementioned problems are initial value problems or initial boundary value problem with Dirichlet boundary conditions. In this paper, we consider the heat equations with nonlinear dynamical boundary conditions and according to our best knowledge there are no results about the SWR algorithm for this type of problems. This type of boundary conditions arises for many known equations of mathematical physics. They are motivated by problems in diffusion phenomena [17], reaction diffusion systems in phase-transition phenomena [18], models in climatology [19], and many others. For physical interpretations of dynamical boundary conditions for problem (1), the interested reader can refer to [20].

Here, we focus on the convergence properties of the algorithm on sufficient long time intervals and the dependence of the convergence rate on the overlap size and the nonlinearity of the boundary nonlinear functions. The remainder of this paper is organized as follows. In Section 2, we introduce 
the problem studied in this paper and the SWR iterative formula for this problem. Our main results are presented in Section 3, where we prove convergence of the SWR algorithm and investigate how the convergence rate depends on the overlap size and the nonlinearity of the boundary nonlinear functions. Section 4 provides some numerical experiments to validate our theoretical finding. Finally, in Section 5 we finish this paper by some conclusion remarks.

\section{Problem Description and the SWR Algorithm}

We consider the following problem on space domain $\Omega=$ $[0,1]$ :

$$
\begin{gathered}
\partial_{t} u=\partial_{x x} u+f(x, t), \quad(x, t) \in \Omega \times \mathbb{R}^{+}, \\
u(x, 0)=u_{0}(x), \quad x \in \Omega, \\
\partial_{x} u(0, t)=g_{1}(u(0, t), t), \quad t \in \mathbb{R}^{+}, \\
\partial_{x} u(1, t)=g_{2}(u(1, t), t), \quad t \in \mathbb{R}^{+},
\end{gathered}
$$

where $f(x, t) \in \mathbb{C}\left(\Omega ; \mathbb{R}^{+}\right), g_{1,2}$ are smooth functions and satisfying growth assumption

$$
\lim _{u \rightarrow+\infty}\left(\inf \frac{g_{1,2}(u, t)}{u}\right)<+\infty .
$$

With this assumption, we know from Corollary 3.6 in [21] that the solution of (1) is globally defined and bounded. We decompose $\Omega=(0,1)$ into two overlapping subdomains $\Omega=\Omega_{1} \cup \Omega_{2}$, with $\Omega_{1}=(0, \beta)$ and $\Omega_{2}=(\alpha, 1)$ and $0<\alpha \leq \beta<1$. Following the spirit of [5,6], the twosubdomain SWR algorithm for (1) can be written as

$$
\begin{gathered}
\partial_{t} u_{1}^{k}=\partial_{x x} u_{1}^{k}+f(x, t), \quad(x, t) \in \Omega_{1} \times \mathbb{R}^{+}, \\
u_{1}^{k}(x, 0)=u_{0}(x), \quad x \in \Omega_{1}, \\
\partial_{x} u_{1}^{k}(0, t)=g_{1}\left(u_{1}^{k}(0, t), t\right), \quad t \in \mathbb{R}^{+}, \\
u_{1}^{k}(\beta, t)=u_{2}^{k-1}(\beta, t), \quad t \in \mathbb{R}^{+}, \\
\partial_{t} u_{2}^{k}=\partial_{x x} u_{2}^{k}+f(x, t), \quad(x, t) \in \Omega_{2} \times \mathbb{R}^{+}, \\
u_{2}^{k}(x, 0)=u_{0}(x), \quad x \in \Omega_{2}, \\
u_{2}^{k}(\alpha, t)=u_{1}^{k-1}(\alpha, t), \quad t \in \mathbb{R}^{+}, \\
\partial_{x} u_{2}^{k}(1, t)=g_{2}\left(u_{2}^{k}(1, t), t\right), \quad t \in \mathbb{R}^{+},
\end{gathered}
$$

where $k \geq 1$ is the iteration index and $u_{1,2}^{0}$ are initial approximations to the solution $u$ of (1).

The comparison principle is central for the convergence analysis of the overlapping Schwarz waveform relaxation algorithm. For the Dirichlet type boundary conditions, this principle is known for long and can be found in every textbook concerning PDEs (see, e.g., [22]) and has been used widely in the SWR community $[5,6,8,12]$. The comparison principle for dynamical boundary conditions is however much less known, until recently by von Below and de Coster [23]. Rault [24, 25], von Below and Pincet Mailly [26], and Pincet Mailly [27] used this comparison principle to study the blow-up phenomenon for strong nonlinear reaction diffusion equations.

Lemma 1. Let $d_{1,2}>0, \widetilde{\Omega}=(a, b) \subseteq(0,1)$, and the function $U$ satisfy the following differential inequalities

$$
\begin{gathered}
\partial_{t} U-\partial_{x x} U \geq 0, \quad(x, t) \in \widetilde{\Omega} \times \mathbb{R}^{+}, \\
U(x, 0) \geq 0, \quad x \in \widetilde{\Omega}, \\
\partial_{x} U(a, t)+d_{1} U(a, t) \geq 0, \quad t \in \mathbb{R}^{+}, \\
\partial_{x} U(b, t)+d_{2} U(b, t) \geq 0, \quad t \in \mathbb{R}^{+} .
\end{gathered}
$$

Then, it holds that $U(x, t) \geq 0$, for all $x \in[a, b]$ and $t \geq 0$.

Proof. See Theorem 2.2 in [23].

Note that, Lemma 1 can be also used to hybrid boundary conditions. For example, the left boundary condition in (4) can be equivalently rewritten as $\left(1 / d_{1}\right) \partial_{x} U(a, t)+U(a, t) \geq$ 0 and thus by letting $d_{1} \rightarrow+\infty$ we can also conclude that $U(x, t) \geq 0$ from (4) by imposing $U(a, t) \geq 0$ instead of the dynamical boundary condition.

\section{Convergence Analysis}

In this section, we analyze the convergence of the SWR iteration (3). To this end, we define $e_{j}^{k}=u_{j}-u_{j}^{k}$ with $u_{j}=$ $\left.u\right|_{x \in \Omega_{j}}, j=1,2$. Subtracting (3) from (1) gives

$$
\begin{gathered}
\partial_{t} e_{1}^{k}-\partial_{x x} e_{1}^{k}=0, \quad(x, t) \in \Omega_{1} \times \mathbb{R}^{+}, \\
e_{1}^{k}(x, 0)=0, \quad x \in \Omega_{1}, \\
\partial_{x} e_{1}^{k}(0, t)=g_{1}\left(u_{1}(0, t), t\right)-g_{1}\left(u_{1}^{k}(0, t), t\right), \quad t \in \mathbb{R}^{+}, \\
e_{1}^{k}(\beta, t)=e_{2}^{k-1}(\beta, t), \quad t \in \mathbb{R}^{+}, \\
\partial_{t} e_{2}^{k}-\partial_{x x} e_{2}^{k}=0, \quad(x, t) \in \Omega_{2} \times \mathbb{R}^{+}, \\
e_{2}^{k}(x, 0)=0, \quad x \in \Omega_{2}, \\
e_{2}^{k}(\alpha, t)=e_{1}^{k-1}(\alpha, t), \quad t \in \mathbb{R}^{+}, \\
\partial_{x} e_{2}^{k}(1, t)=g_{2}\left(u_{2}(1, t), t\right)-g_{2}\left(u_{2}^{k}(1, t), t\right), \quad t \in \mathbb{R}^{+} .
\end{gathered}
$$

By using the Taylors' expansion we get

$$
\begin{gathered}
\partial_{t} e_{1}^{k}-\partial_{x x} e_{1}^{k}=0, \quad(x, t) \in \Omega_{1} \times \mathbb{R}^{+}, \\
e_{1}^{k}(x, 0)=0, \quad x \in \Omega_{1}, \\
\partial_{x} e_{1}^{k}(0, t)-d_{1}\left(\tilde{u}_{1}^{k}, t\right) e_{1}^{k}(0, t)=0, \quad t \in \mathbb{R}^{+},
\end{gathered}
$$




$$
\begin{gathered}
e_{1}^{k}(\beta, t)=e_{2}^{k-1}(\beta, t), \quad t \in \mathbb{R}^{+}, \\
\partial_{t} e_{2}^{k}-\partial_{x x} e_{2}^{k}=0, \quad(x, t) \in \Omega_{2} \times \mathbb{R}^{+}, \\
e_{2}^{k}(x, 0)=0, \quad x \in \Omega_{2}, \\
e_{2}^{k}(\alpha, t)=e_{1}^{k-1}(\alpha, t), \quad t \in \mathbb{R}^{+}, \\
\partial_{x} e_{2}^{k}(1, t)-d_{2}\left(\widetilde{u}_{2}^{k}, t\right) e_{2}^{k}(1, t)=0, \quad t \in \mathbb{R}^{+},
\end{gathered}
$$

where $\tilde{u}_{1,2}^{k}$ are functions of $x$ and $t$ which lie between $u_{1,2}$ and $u_{1,2}^{k}$ and $d_{1,2}(u, t)$ are defined by

$$
d_{1,2}(u, t)=\partial_{u} g_{1,2}(u, t)
$$

For any function $f \in L^{\infty}\left(\widetilde{\Omega} ; \mathbb{R}^{+}\right)($where $\widetilde{\Omega} \subset \mathbb{R})$, we define

$$
\|f(x, \cdot)\|_{\infty}=\sup _{t>0}|f(x, t)|, \quad\|f\|_{\infty, \infty}=\sup _{x \in \widetilde{\Omega}, t>0}|f(x, t)| .
$$

Theorem 2. Assume that $0<\alpha<\beta<1$ and that the nonlinear boundary functions $g_{1,2}$ satisfy

$$
\partial_{u} g_{1,2}(u, t) \leq \mu_{1,2}<0, \quad\left(\text { i.e., } d_{1,2} \leq \mu_{1,2}<0\right),
$$

where $\mu_{1,2}$ are constants. Then the error functions $e_{1,2}^{k}$ of the SWR iteration (3) decay linearly to zero. Specifically, it holds that

$$
\begin{aligned}
& \left\|e_{1}^{2 k+1}\right\|_{\infty, \infty} \leq \rho^{k}\left\|e_{2}^{0}(\alpha, \cdot)\right\|_{\infty}, \\
& \left\|e_{2}^{2 k+1}\right\|_{\infty, \infty} \leq \rho^{k}\left\|e_{1}^{0}(\beta, \cdot)\right\|_{\infty},
\end{aligned}
$$

where $\rho$ is the called the convergence factor and is defined by

$$
\rho=\frac{\left(1-\mu_{1} \alpha\right)\left[\mu_{2}(\beta-1)+1\right]}{\left(1-\mu_{1} \beta\right)\left[\mu_{2}(\alpha-1)+1\right]} .
$$

Proof. Consider the following differential equations:

$$
\begin{gathered}
\partial_{t} \bar{e}_{1}^{k}-\partial_{x x} \bar{e}_{1}^{k}=0, \quad(x, t) \in \Omega_{1} \times \mathbb{R}^{+}, \\
\bar{e}_{1}^{k}(x, 0)=\frac{1-\mu_{1} x}{1-\mu_{1} \beta}\left\|e_{2}^{k-1}(\beta, \cdot)\right\|_{\infty}, \quad x \in \Omega_{1}, \\
\partial_{x} \bar{e}_{1}^{k}(0, t)-\mu_{1} \bar{e}_{1}^{k}(0, t)=\frac{-2 \mu_{1}}{1-\mu_{1} \beta}\left\|e_{2}^{k-1}(\beta, \cdot)\right\|_{\infty}, \quad t \in \mathbb{R}^{+}, \\
\bar{e}_{1}^{k}(\beta, t)=\left\|e_{2}^{k-1}(\beta, \cdot)\right\|_{\infty}, \quad t \in \mathbb{R}^{+}, \\
\partial_{t} \bar{e}_{2}^{k}-\partial_{x x} \bar{e}_{2}^{k}=0, \quad(x, t) \in \Omega_{2} \times \mathbb{R}^{+}, \\
\bar{e}_{2}^{k}(x, 0)=\frac{\mu_{2}(x-1)+1}{1+\mu_{2}(\alpha-1)}\left\|e_{1}^{k-1}(\alpha, \cdot)\right\|_{\infty}, \quad x \in \Omega_{2}, \\
\bar{e}_{2}^{k}(\alpha, t)=\left\|e_{1}^{k-1}(\alpha, \cdot)\right\|_{\infty}, \quad t \in \mathbb{R}^{+}, \\
\partial_{x} \bar{e}_{2}^{k}(1, t)-\mu_{2} \bar{e}_{2}^{k}(1, t)=0, \quad t \in \mathbb{R}^{+} .
\end{gathered}
$$

It is easy to find that the solutions to (13) are the steady state solutions

$$
\begin{gathered}
\bar{e}_{1}^{k}(x, t)=\frac{1-\mu_{1} x}{1-\mu_{1} \beta}\left\|e_{2}^{k-1}(\beta, \cdot)\right\|_{\infty}, \quad x \in[0, \beta], \\
\bar{e}_{2}^{k}(x, t)=\frac{\mu_{2}(x-1)+1}{\mu_{2}(\alpha-1)+1}\left\|e_{1}^{k-1}(\alpha, \cdot)\right\|_{\infty}, \quad x \in[\alpha, 1] .
\end{gathered}
$$

Since $\mu_{1,2}<0$, it is easy to know that $\bar{e}_{1}^{k}(x, t) \geq 0$ for all $(x, t) \epsilon$ $[0, \beta] \times[0,+\infty)$ and $\bar{e}_{2}^{k}(x, t) \geq 0$ for all $(x, t) \in[\alpha, 1] \times[0,+\infty)$.

Now, let $E_{j}^{k}=\bar{e}_{j}^{k}-e_{j}^{k}$ and then by subtracting (6) from (13) we get

$$
\begin{gathered}
\partial_{t} E_{1}^{k}-\partial_{x x} E_{1}^{k}=0, \quad(x, t) \in \Omega_{1} \times \mathbb{R}^{+}, \\
E_{1}^{k}(x, 0) \geq 0, \quad x \in \Omega_{1},
\end{gathered}
$$

$\partial_{x} E_{1}^{k}(0, t)-d_{1} E_{1}^{k}(0, t)=\left(\mu_{1}-d_{1}\right) \bar{e}_{1}(0, t)$

$$
+\frac{-2 \mu_{1}}{1-\mu_{1} \beta}\left\|e_{2}^{k-1}(\beta, \cdot)\right\|_{\infty}, \quad t \in \mathbb{R}^{+},
$$

$$
\begin{gathered}
E_{1}^{k}(\beta, t) \geq 0, \quad t \in \mathbb{R}^{+}, \\
\partial_{t} E_{2}^{k}-\partial_{x x} E_{2}^{k}=0, \quad(x, t) \in \Omega_{2} \times \mathbb{R}^{+}, \\
E_{2}^{k}(x, 0) \geq 0, \quad x \in \Omega_{2}, \\
E_{2}^{k}(\alpha, t) \geq 0, \quad t \in \mathbb{R}^{+}, \\
\partial_{x} E_{2}^{k}(1, t)-d_{2} E_{2}^{k}(1, t)=\left(\mu_{2}-d_{2}\right) \bar{e}_{2}(1, t), \quad t \in \mathbb{R}^{+} .
\end{gathered}
$$

By using $d_{1,2} \leq \mu_{1,2}<0$ and $\bar{e}_{1,2}(x, t) \geq 0$, we have $\partial_{x} E_{1}^{k}(0, t)-$ $d_{1} E_{1}^{k}(0, t) \geq 0$ and $\partial_{x} E_{2}^{k}(1, t)-d_{2} E_{2}^{k}(1, t) \geq 0$. Therefore, it follows by applying Lemma 1 that $E_{j}^{k} \geq 0$; that is, $e_{j}^{k} \leq \bar{e}_{j}^{k}$ $(j=1,2)$. Similarly, the sum $\widehat{E}_{j}^{k}:=e_{j}^{k}+\bar{e}_{j}^{k}$ are also nonnegative functions and thus the modulus of $e_{j}^{k}$ can be bounded by

$$
\begin{gathered}
\left|e_{1}^{k}(x, t)\right| \leq \frac{1-\mu_{1} x}{1-\mu_{1} \beta}\left\|e_{2}^{k-1}(\beta, \cdot)\right\|_{\infty}, \quad x \in[0, \beta], t \geq 0, \\
\left|e_{2}^{k}(x, t)\right| \leq \frac{\mu_{2}(x-1)+1}{\mu_{2}(\alpha-1)+1}\left\|e_{1}^{k-1}(\alpha, \cdot)\right\|_{\infty}, \quad x \in[\alpha, 1], t \geq 0,
\end{gathered}
$$

and this implies that

$$
\begin{gathered}
\left\|e_{1}^{k}(x, \cdot)\right\| \leq \frac{1-\mu_{1} x}{1-\mu_{1} \beta}\left\|e_{2}^{k-1}(\beta, \cdot)\right\|_{\infty}, \quad x \in[0, \beta], \\
\left\|e_{2}^{k}(x, \cdot)\right\| \leq \frac{\mu_{2}(x-1)+1}{\mu_{2}(\alpha-1)+1}\left\|e_{1}^{k-1}(\alpha, \cdot)\right\|_{\infty}, \quad x \in[\alpha, 1] .
\end{gathered}
$$

From (17) we get

$$
\begin{gathered}
\left\|e_{1}^{k}(\alpha, \cdot)\right\| \leq \frac{1-\mu_{1} \alpha}{1-\mu_{1} \beta}\left\|e_{2}^{k-1}(\beta, \cdot)\right\|_{\infty} \\
\left\|e_{2}^{k}(\beta, \cdot)\right\| \leq \frac{\mu_{2}(\beta-1)+1}{\mu_{2}(\alpha-1)+1}\left\|e_{1}^{k-1}(\alpha, \cdot)\right\|_{\infty} .
\end{gathered}
$$


Then, by a simple recursion it can be shown that

$$
\left\|e_{1}^{2 k}(\alpha, \cdot)\right\| \leq \rho^{k}\left\|e_{1}^{0}(\alpha, \cdot)\right\|_{\infty}, \quad\left\|e_{2}^{2 k}(\beta, \cdot)\right\| \leq \rho^{k}\left\|e_{2}^{0}(\beta, \cdot)\right\|_{\infty},
$$

where the argument $\rho$ is defined by (11). Now, substituting (19) into (17) and taking supremum with respect to $x$ in each inequality gives (10). For the vanishing of the errors $e_{j}^{k}$, it just needs to notice $\rho<1$ because $\mu_{1,2}<0$ and $0<\alpha<\beta<1$.

Remark 3. Let $s=\beta-\alpha>0$ be the overlap size. Then, by substituting $\beta=s+\alpha$ into (11) we have

$$
\rho=\frac{\left(1-\mu_{1} \alpha\right)\left[\mu_{2}(s+\alpha-1)+1\right]}{\left(1-\mu_{1}[s+\alpha]\right)\left[\mu_{2}(\alpha-1)+1\right]} .
$$

It is easy to get

$$
\begin{aligned}
& \partial_{s} \rho=\frac{\left(1-\mu_{1} \alpha\right)}{\mu_{2}(\alpha-1)+1} \times \frac{\mu_{1}+\mu_{2}-\mu_{1} \mu_{2}}{\left[1-\mu_{1}(s+\alpha)\right]^{2}}<0, \\
& \partial_{\mu_{1}} \rho=\frac{s\left[\mu_{2}(s+\alpha-1)+1\right]}{\left(1-\mu_{1}[s+\alpha]\right)^{2}\left[\mu_{2}(\alpha-1)+1\right]}>0, \\
& \partial_{\mu_{2}} \rho=\frac{s\left(1-\mu_{1} \alpha\right)}{\left(1-\mu_{1}[s+\alpha]\right)\left[\mu_{2}(\alpha-1)+1\right]^{2}}>0 .
\end{aligned}
$$

Hence, increasing $s$ (i.e., increasing the overlap size) will improve the convergence rate of the SWR algorithm. Moreover, the other two derivatives given in (21) imply that the SWR algorithm (3) is especially suitable for problem (1) that the nonlinear boundary functions have very large negative partial derivatives. The limiting case $\mu_{1}, \mu_{2} \rightarrow-\infty$ yields

$$
\rho=\frac{\alpha(1-\beta)}{\beta(1-\alpha)},
$$

which is the convergence factor of the two-subdomain SWR algorithm for the heat equation (1) with Dirichlet boundary condition (see [5]).

\section{Numerical Results}

In this section, we present some numerical results to validate our theoretical predictions obtained in Section 3. For (1), we impose initial condition $u(x, 0)=0$. The source function $f(x, t)$ is chosen as

$$
f(x, t)=\partial_{t} \tilde{u}(x, t)-\partial_{x x} \widetilde{u}(x, t),
$$

where $\tilde{u}(x, t)=\sin ^{1999}(4 \pi x(1-x)(\sqrt{t} /(1+t)))$. The nonlinear boundary functions are chosen as

$$
\begin{gathered}
g_{1}(u, t)=a u+\sin \left(\sqrt{t} e^{t}\right)^{2} u^{3} \\
g_{2}(u, t)=a u+2 \cos \left(2 \cos \left(t e^{\sqrt{t}}\right) u\right) .
\end{gathered}
$$

With the above choice, it is easy to know that the exact solution to (1) is $u(x, t)=\widetilde{u}(x, t)$. We plot the exact solution $\tilde{u}(x, t)$ in Figure 2(a), where we can see that the exact solution is almost zero except around " $x=0.5$ " due to the power "1999." The reason why we choose this function as the reference solution is that it is easy to intuitively see the evolution of the iterates $u_{j}^{k}$ on subdomain $x \in \Omega_{j}$ during iterations, as shown in Figure 1. We equally decompose the space domain into two subdomains with overlap size $s=$ $m \Delta x$, where $m \geq 1$ is an integer and $\Delta x$ is the space mesh size. Then, for each subproblem we discretize the governing equation by using a central finite difference in space with mesh parameter $\Delta x=0.025$ and a backward Euler method in time with time step $\Delta t=0.025$. The space partial derivatives appearing in boundaries are discretized by the upwind finite difference method. All of our experiments are performed in the time interval $t \in[0,30]$.

In this first set of experiments, we investigate the influence of the overlap size $s$ on the convergence rate of the SWR algorithm. We choose $a=30$ in (24) and then we show in Figure 1 the solution generated by the SWR algorithm after 2, 4, and 6 iterations with two different overlap sizes $s=4 \Delta x$ (top) and $s=8 \Delta x$ (bottom). Here, we just show the solution profile on the space-time domain $(x, t) \epsilon$ $[0,1] \times[0,3]$ for better viewing purpose. The converged solution is plotted in Figure 2 on the left. We can see clearly that the overlap size has a remarkable influence on the convergence rate and in particular with a larger overlap size the algorithm converges faster. This observation confirms our theoretical predication given in Section 3. The measured error (between the converged solution and the iterates, in the sense of $L^{\infty}$ norm) together with the linear bound predicted by Theorem 2 is shown in Figure 2 on the right. We see that the theoretical linear bound predicts the actual convergence rate very well.

Next, we study how the parameter $a$ in (24) affects the convergence rate of the SWR algorithm. Note that, with the nonlinear boundary functions (24), the arguments $\mu_{1}$ and $\mu_{2}$ appearing in the convergence factor $\rho$ are $a+3$ and $a+4$, respectively. We choose three different $a$ and plot the measured error in Figure 3. We see that decreasing $a$ has a positive effect on the convergence rate, which confirms our theoretical analysis done in Remark 3.

\section{Conclusion}

We have briefly analyzed the convergence properties of the overlapping SWR algorithm for heat equation with nonlinear dynamical boundary conditions, based on the recently established comparison principle for PDEs with dynamical boundary conditions [23]. The linear convergence on sufficient long time intervals is proved and it is shown that the convergence rate ameliorates as one increases the overlap size. The dependence of the convergence rate on the nonlinearity of the nonlinear boundary functions is also revealed.

Further work includes generalizing the current work to many subdomains for practical parallel computation purpose. Also, to speed up the convergence rate dynamical transmission conditions, such as the popular Robin transmission conditions, should be utilized. Note that the transmission 

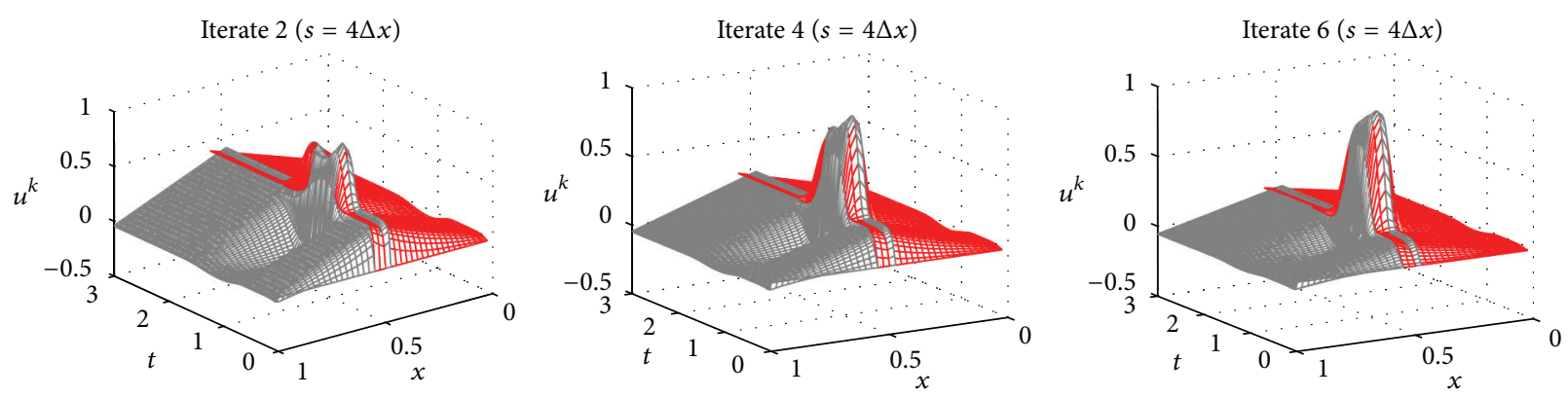

(a)
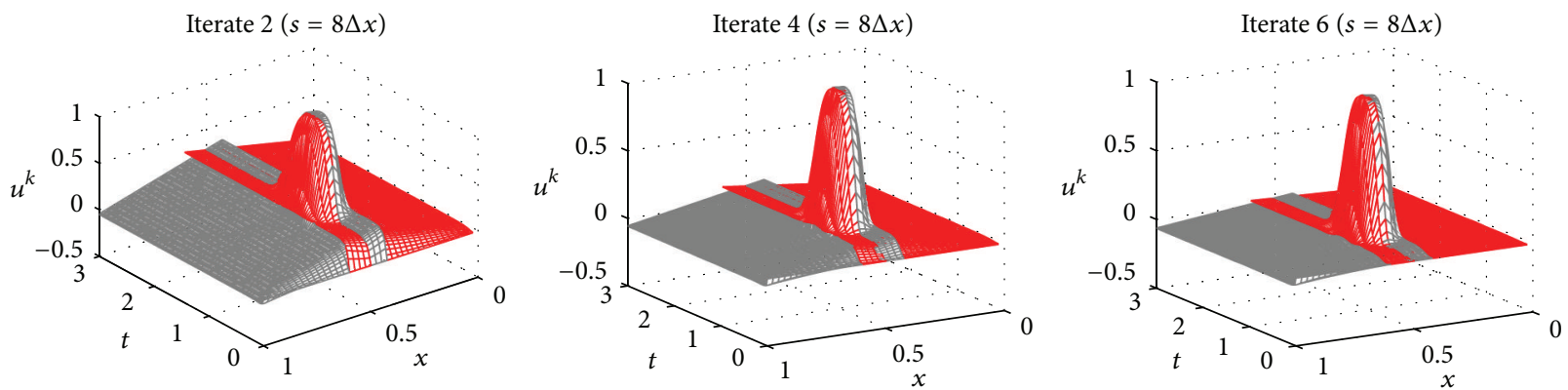

(b)

FIGURE 1: Influence of the overlap size on the convergence rate of the SWR algorithm. Top: with $s=4 \Delta x$ the solution generated by the SWR algorithm after 2, 4, and 6 iterations; bottom: the similar information for $s=8 \Delta x$.

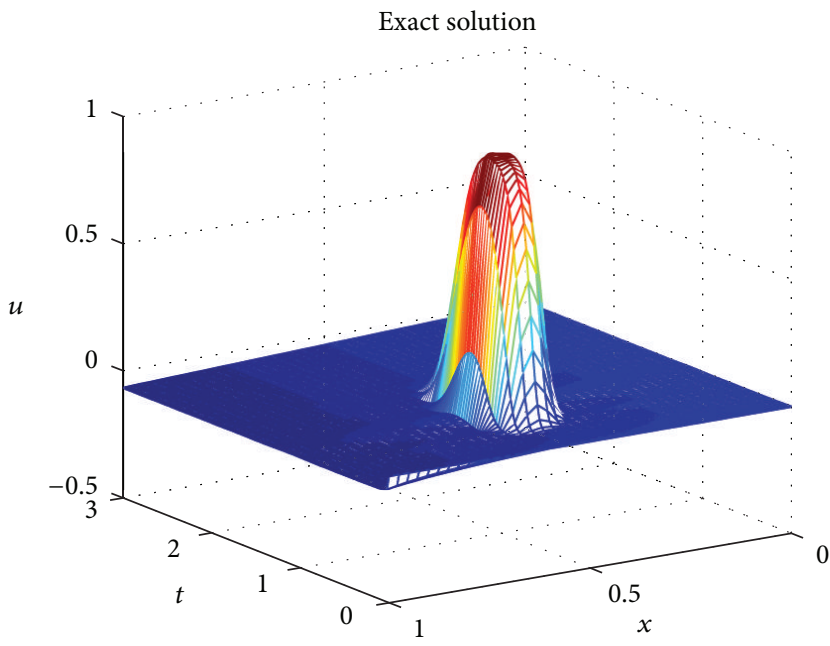

(a)

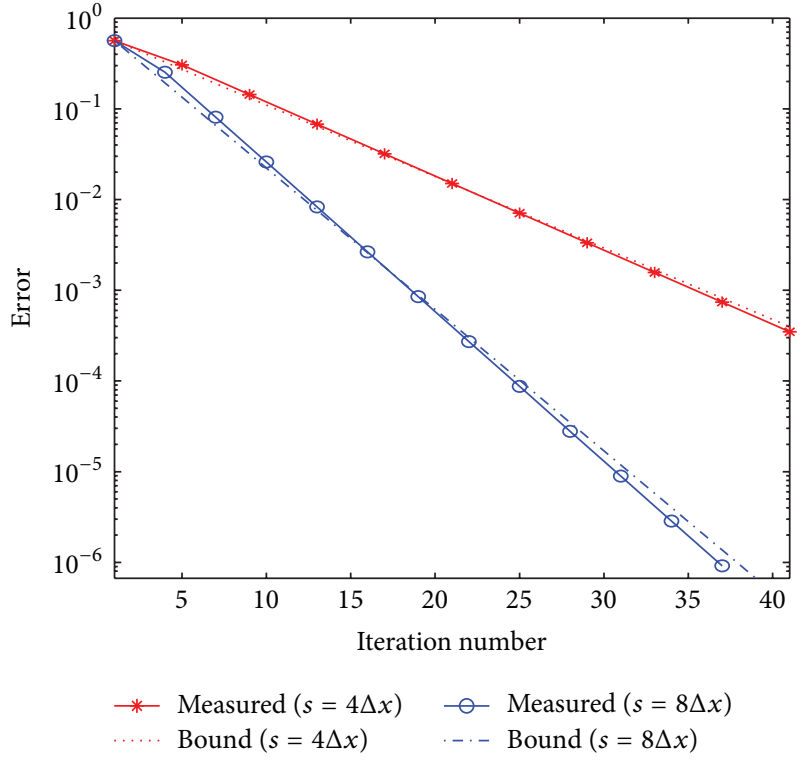

(b)

Figure 2: (a) The profile of $u(x, t)$ on domain $(x, t) \in[0,1] \times[0,3]$. (b) Convergence rate together with the theoretical linear bound of the SWR algorithm with two different overlap sizes.

conditions used in this paper are Dirichlet type. For time dependent PDEs (initial problems on unbounded space domain), it is well known that the dynamical transmission conditions always yield significant convergence rate for the SWR algorithm (see, e.g., $[9,10]$ ). Due to the nonlinearity arising from the boundaries and the boundedness of the space domain, it is impossible to straightforwardly generalize the techniques proposed for Dirichlet boundary conditions to dynamical boundary conditions and new notion is eagerly expected. 


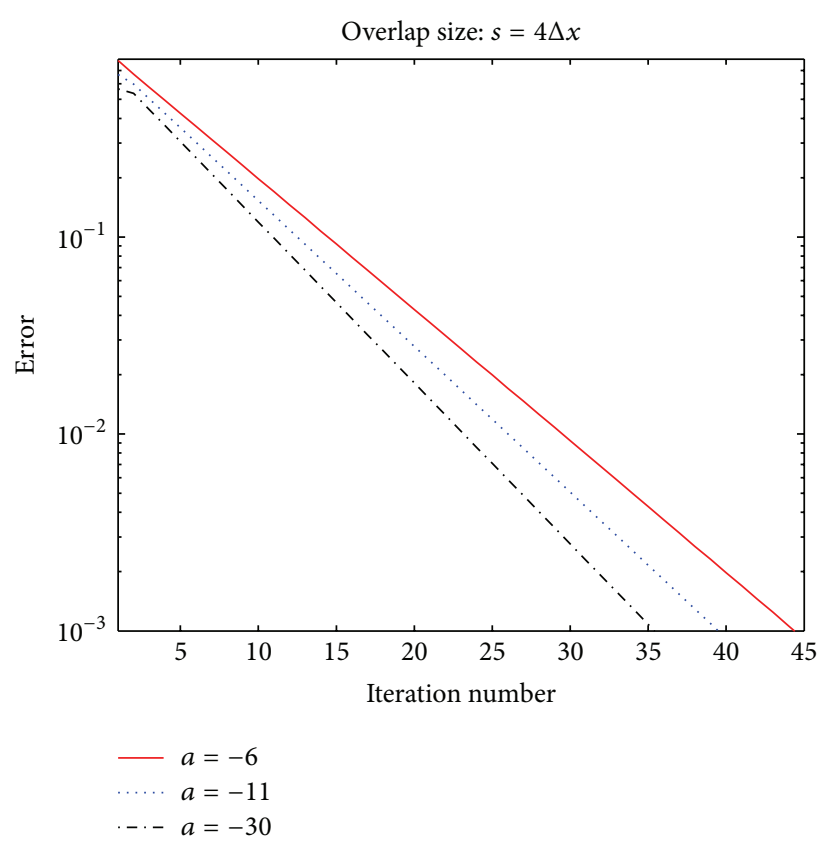

(a)

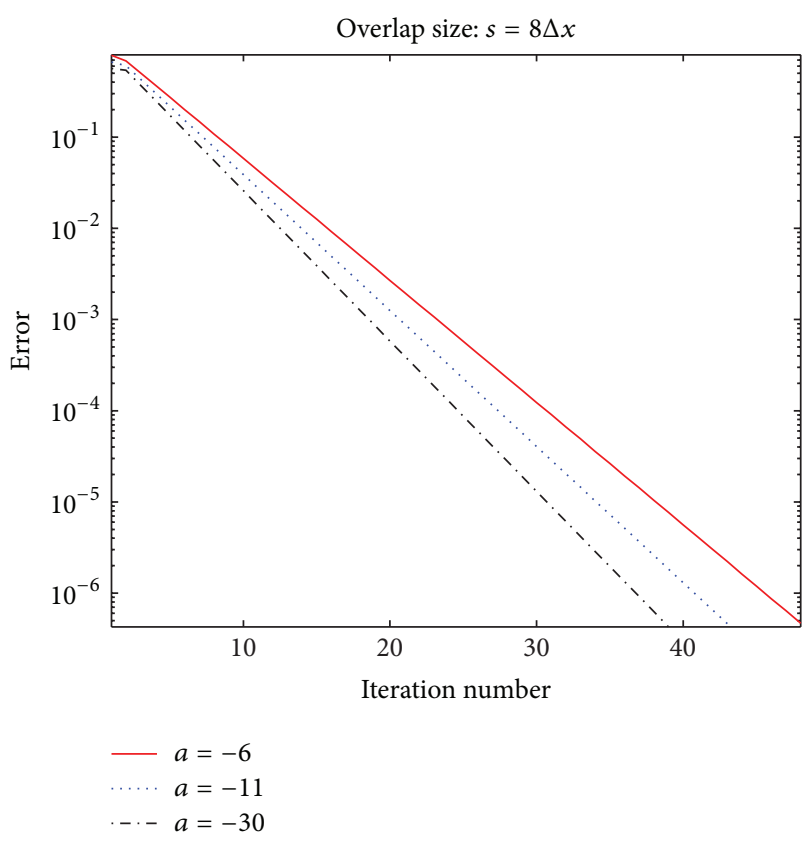

(b)

FIGURE 3: Influence of the problem parameter $a$ on the convergence rate of the SWR algorithm. (a) $s=4 \Delta x$; (b) $s=8 \Delta x$.

\section{Acknowledgments}

The authors are very grateful to the anonymous referees for the careful reading of a preliminary version of the paper and their valuable suggestions and comments, which really improve the quality of this paper. This work was supported by the NSF of Sichuan University of Science and Engineering (2011RZY04 and 2012RC24), the project of the Key Laboratory of Cambridge and Non-Destructive Inspection of Sichuan Institutes of Higher Education (2013QZY01), and the NSF of China (11301362, 11226312, 11371157, and 91130003).

\section{References}

[1] T. P. A. Mathew, Domain Decomposition Methods for the Numerical Solution of Partial Differential Equations, vol. 61, Springer, Berlin, Germany, 2008.

[2] A. Toselli and O. Widlund, Domain decomposition methodsalgorithms and theory, vol. 34, Springer, Berlin, Germany, 2005.

[3] E. Lelarasmee, A. E. Ruehli, and A. L. Sangiovanni-Vincentelli, "Waveform relaxation method for time-domain analysis of large scale integrated circuits," IEEE Transactions on ComputerAided Design of Integrated Circuits and Systems, vol. 1, no. 3, pp. 131-145, 1983.

[4] Y.-L. Jiang, "On time-domain simulation of lossless transmission lines with nonlinear terminations," SIAM Journal on Numerical Analysis, vol. 42, no. 3, pp. 1018-1031, 2004.

[5] M. J. Gander and A. M. Stuart, "Space-time continuous analysis of waveform relaxation for the heat equation," SIAM Journal on Scientific Computing, vol. 19, no. 6, pp. 2014-2031, 1998.

[6] M. J. Gander, "A waveform relaxation algorithm with overlapping splitting for reaction diffusion equations," Numerical Linear Algebra with Applications, vol. 6, no. 2, pp. 125-145, 1999.
[7] E. Giladi and H. B. Keller, "Space-time domain decomposition for parabolic problems," Numerische Mathematik, vol. 93, no. 2, pp. 279-313, 2002.

[8] M. J. Gander and H. Zhao, "Overlapping Schwarz waveform relaxation for the heat equation in $n$ dimensions," BIT Numerical Mathematics, vol. 42, no. 4, pp. 779-795, 2002.

[9] D. Bennequin, M. J. Gander, and L. Halpern, "A homographic best approximation problem with application to optimized Schwarz waveform relaxation," Mathematics of Computation, vol. 78, no. 265, pp. 185-223, 2009.

[10] M. J. Gander and L. Halpern, "Optimized Schwarz waveform relaxation methods for advection reaction diffusion problems," SIAM Journal on Numerical Analysis, vol. 45, no. 2, pp. 666-697, 2007.

[11] V. Martin, "An optimized Schwarz waveform relaxation method for the unsteady convection diffusion equation in two dimensions," Applied Numerical Mathematics, vol. 52, no. 4, pp. 401428, 2005.

[12] M. J. Gander and C. Rohde, "Overlapping Schwarz waveform relaxation for convection-dominated nonlinear conservation laws," SIAM Journal on Scientific Computing, vol. 27, no. 2, pp. 415-439, 2005.

[13] V. Martin, "Schwarz waveform relaxation algorithms for the linear viscous equatorial shallow water equations," SIAM Journal on Scientific Computing, vol. 31, no. 5, pp. 3595-3625, 2009.

[14] V. Dolean, M. J. Gander, and L. Gerardo-Giorda, "Optimized Schwarz methods for Maxwell's equations," SIAM Journal on Scientific Computing, vol. 31, no. 3, pp. 2193-2213, 2009.

[15] M. El Bouajaji, V. Dolean, M. J. Gander, and S. Lanteri, "Optimized Schwarz methods for the time-harmonic Maxwell equations with damping," SIAM Journal on Scientific Computing, vol. 34, no. 4, pp. A2048-A2071, 2012. 
[16] H. Zhang and Y. L. Jiang, "Schwarz waveform relaxation methods of parabolic time-periodic problems," Scientia Sinica Mathematica, vol. 5, pp. 497-516, 2010.

[17] M. Warma, "Quasilinear parabolic equations with nonlinear Wentzell-Robin type boundary conditions," Journal of Mathematical Analysis and Applications, vol. 336, no. 2, pp. 1132-1148, 2007.

[18] C. Cavaterra, C. G. Gal, M. Grasselli, and A. Miranville, "Phase-field systems with nonlinear coupling and dynamic boundary conditions," Nonlinear Analysis A: Theory, Methods \& Applications, vol. 72, no. 5, pp. 2375-2399, 2010.

[19] R. G. Watts and M. Morantine, "Rapid climatic change and the deep ocean," Climatic Change, vol. 16, no. 1, pp. 83-97, 1990.

[20] G. R. Goldstein, "Derivation and physical interpretation of general boundary conditions," Advances in Differential Equations, vol. 11, no. 4, pp. 457-480, 2006.

[21] A. Rodríguez-Bernal and A. Tajdine, "Nonlinear balance for reaction-diffusion equations under nonlinear boundary conditions: dissipativity and blow-up," Journal of Differential Equations, vol. 169, no. 2, pp. 332-372, 2001.

[22] C. Pao, Nonlinear Parabolic and Elliptic Equations, Plenum, New York, NY, USA, 1992.

[23] J. von Below and C. de Coster, "A qualitative theory for parabolic problems under dynamical boundary conditions," Journal of Inequalities and Applications, vol. 5, no. 5, pp. 467-486, 2000.

[24] J.-F. Rault, "The Fujita phenomenon in exterior domains under the Robin boundary conditions," Comptes Rendus Mathématique, vol. 349, no. 19-20, pp. 1059-1061, 2011.

[25] J.-F. Rault, "The Fujita phenomenon in exterior domains under dynamical boundary conditions," Asymptotic Analysis, vol. 66, no. 1, pp. 1-8, 2010.

[26] J. von Below and G. Pincet Mailly, "Blow up for reaction diffusion equations under dynamical boundary conditions," Communications in Partial Differential Equations, vol. 28, no. 1-2, pp. 223-247, 2003.

[27] G. Pincet Mailly, "Blow up for nonlinear parabolic equations with time degeneracy under dynamical boundary conditions," Nonlinear Analysis A: Theory, Methods \& Applications, vol. 67, no. 3, pp. 657-667, 2007. 


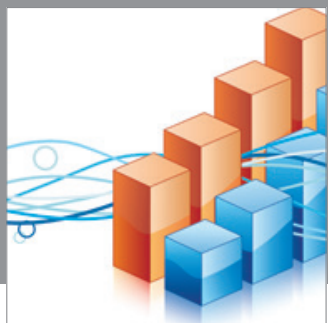

Advances in

Operations Research

mansans

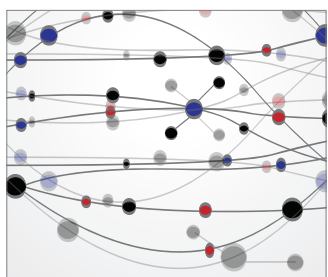

The Scientific World Journal
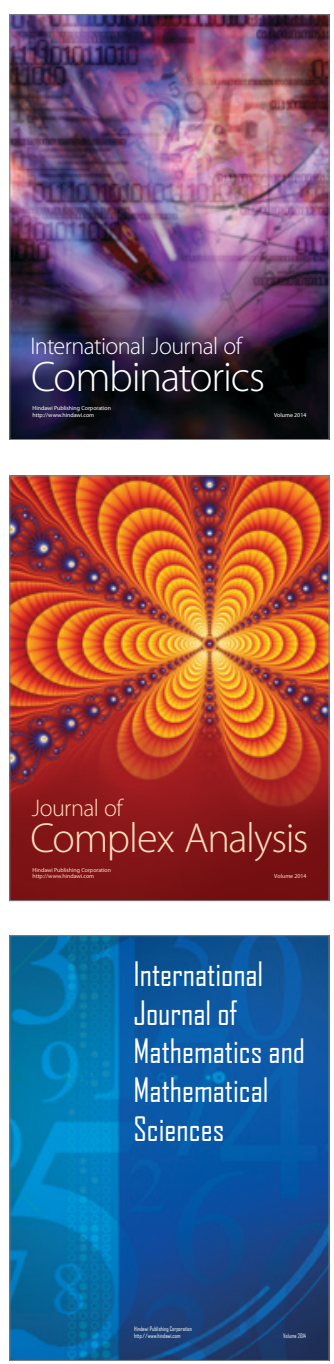
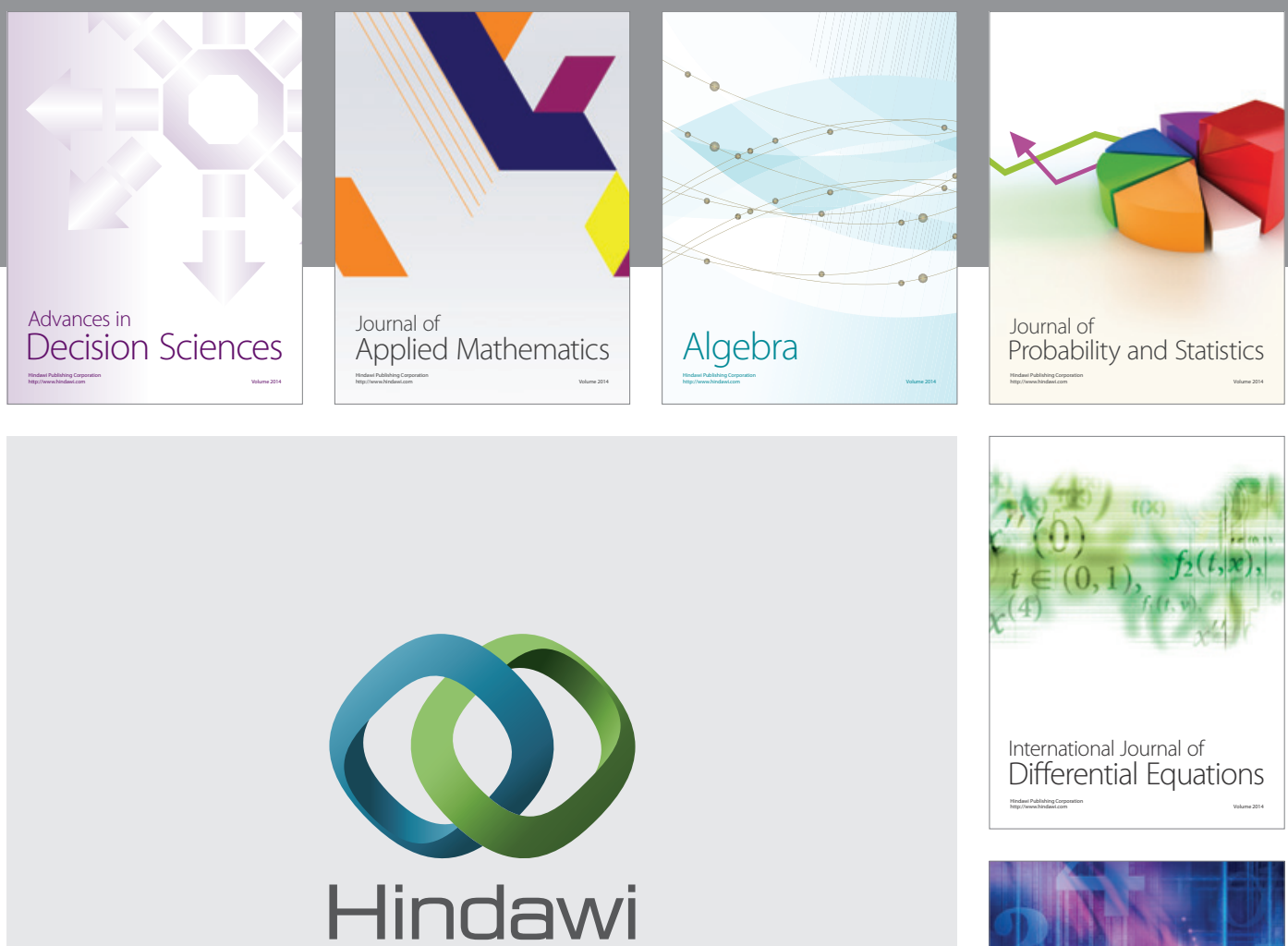

Submit your manuscripts at http://www.hindawi.com
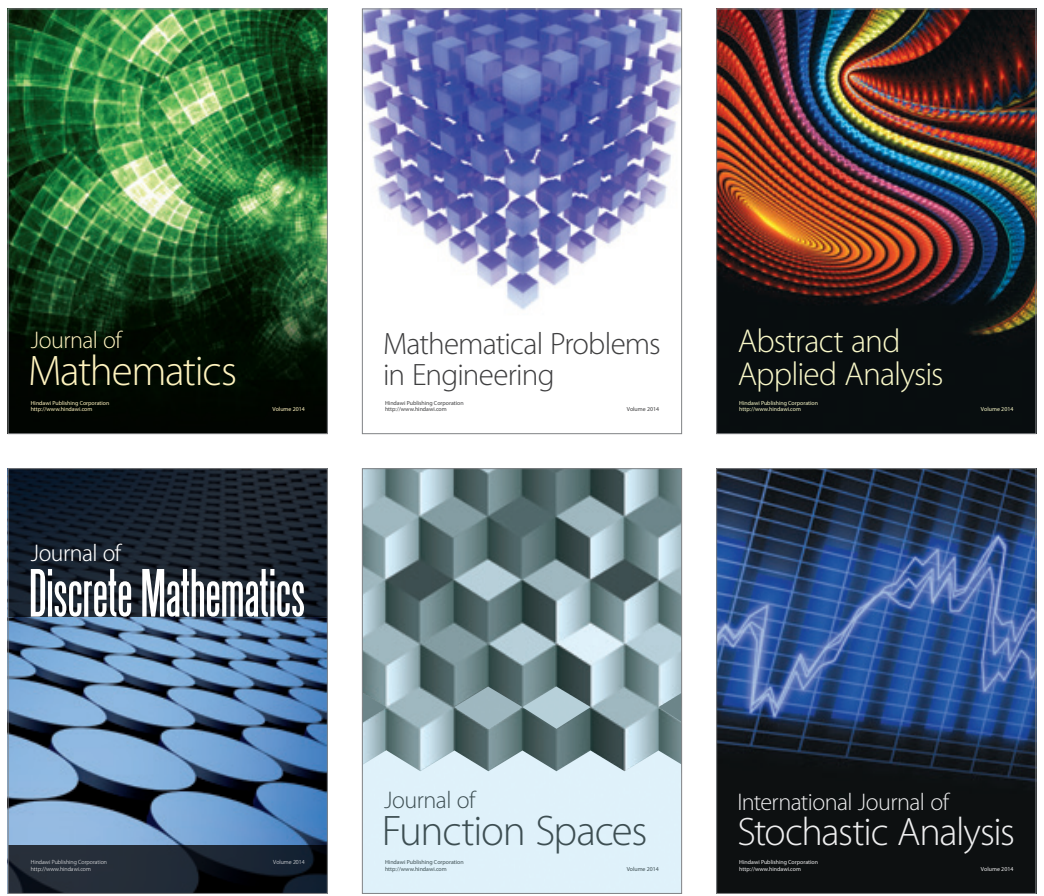

Journal of

Function Spaces

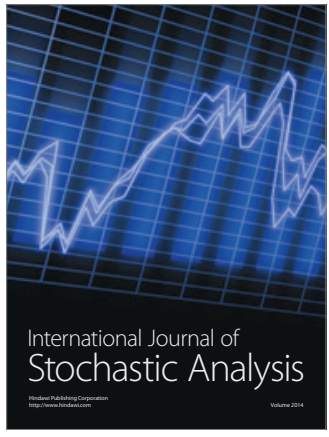

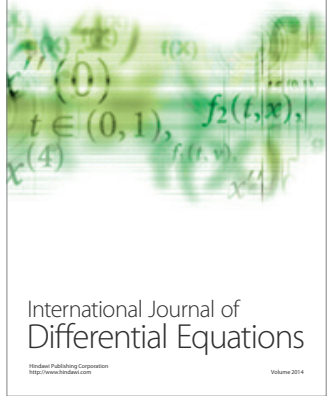
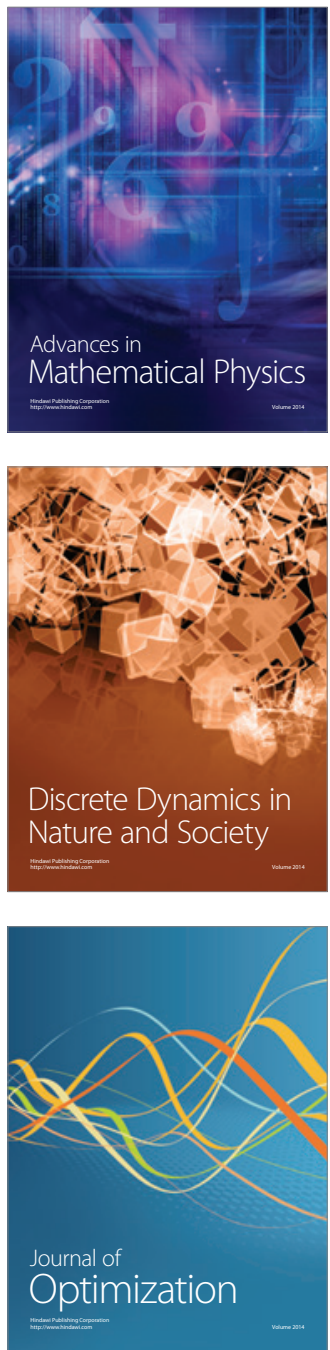